\title{
BMJ Open Gender-specific physical activity-related injuries and risk factors among university students in China: a multicentre population-based cross-sectional study
}

\author{
Weicong Cai (D) , ${ }^{1,2,3}$ Shangmin Chen, ${ }^{1,2}$ Liping Li, ${ }^{1,2}$ Pengying Yue, ${ }^{4}$ Xiaofan $\mathrm{Yu}^{5}$ \\ Lijie Gao, ${ }^{6}$ Wenda Yang, ${ }^{1}$ Cunxian Jia, ${ }^{6}$ Yang Gao (D) ${ }^{7,8}$
}

To cite: Cai W, Chen S, Li L, et al. Gender-specific physical activity-related injuries and risk factors among university students in China: a multicentre population-based crosssectional study. BMJ Open 2020;10:e040865. doi:10.1136/ bmjopen-2020-040865

- Prepublication history and additional material for this paper is available online. To view these files, please visit the journal online (http://dx.doi.org/10. 1136/bmjopen-2020-040865).

Received 25 May 2020 Revised 20 November 2020 Accepted 26 November 2020

Check for updates

(c) Author(s) (or their employer(s)) 2020. Re-use permitted under CC BY-NC. No commercial re-use. See rights and permissions. Published by BMJ.

For numbered affiliations see end of article.

Correspondence to Professor Liping Li; Ipli@stu.edu.cn

\section{ABSTRACT}

Objectives Data on the problem of physical activityrelated injury (PARI) in university students and the risk factors for PARI among different genders are rare. We conducted a multicentre population-based study to investigate the occurrence of PARI and to explore the gender-specific risk factors for PARI among Chinese university students.

Design Cross-sectional study.

Participants A total of 5341 students in grades 1-3 at eight universities in four Chinese cities were selected to complete the online questionnaires during March and April 2017. The questionnaires assessed sociodemographic characteristics, physical activity PA) involvement, sleep duration, sedentary behaviour and PARI experiences in the past 12 months.

Main outcome measures PARI during the past 12 months.

Results Among the 5341 participants, 1293 suffered from at least one PARI in the past 12 months, with an overall incidence rate of $24.2 \%$ (males: $26.2 \%$, females: $23.2 \%$ ) and an injury risk of 0.38 injuries/student/year (males: 0.48 , females: 0.32$)$. Over half of the injured $(57.3 \%)$ experienced a withdrawal time of PA and nearly two-fifths $(39.6 \%)$ required medical attention. Irrespective of gender, Shantou and Xi'an students, sports team members and those who engaged in sports and leisure-time vigorousintensity PA (VPA) at a higher frequency were more likely to suffer from PARI. Male students who participated in sports and leisure-time VPA for long durations had a greater likelihood of sustaining PARI, while having a chronic condition and being involved in sports and leisuretime moderate-intensity PA at a higher frequency and longer duration were potential contributors to PARI among females.

Conclusions The occurrence of PARI and its risk factors differed by gender, which provides a direction towards developing targeted and effective gender-specific preventative programmes to protect Chinese university students from PARI.

\section{INTRODUCTION}

Participation in physical activity (PA) benefits our well-being by lowering the risk of non-communicable diseases, increasing bone
Strengths and limitations of this study

- To our knowledge, this cross-sectional study is the first to explore the gender-specific risk factors associated with injuries resulting from physical activity participation in Chinese university students.

- Data were self-reported, which is subject to reporting bias and recall bias.

- The nature of the cross-sectional study limits us from drawing the cause-and-effect relationship between physical activity-related injury outcome and the potential risk factors.

- Nearly two-thirds of the study samples were female, which may have limited the generalisability and representativeness.

- We did not compare acute and overuse injuries, which may have deviated the association analysis in this study.

density and muscular fitness, delaying the onset of mental diseases and maintaining a healthy weight. ${ }^{1-3}$ In recognition of these well-known benefits of regular PA participation, member states of WHO agreed to a $10 \%$ relative reduction in the prevalence of insufficient PA by $2025 .{ }^{4}$ Meanwhile, there exist several recommendations on PA participation for public health. ${ }^{5-7}$

The promotion of PA participation is a public health priority, however, a potential problem of participation in PA, PA-related injury (PARI), needs to be emphasised, as it has been well documented in different genders or age groups and various types of PAs. ${ }^{8-10}$ Moreover, earlier reports identified PARI as the major health threat to schoolaged adolescents and young adults in many countries. ${ }^{511}$ This means that successful and effective prevention programmes for PARI have great inherent public health gains. ${ }^{12}$ To develop such preventative measures, we first need descriptive injury epidemiology 
describing the characteristics and aetiology of injuries. ${ }^{13}$ Based on previous reports, most injuries occur outdoor and involve lower limbs, and sprains and strains are the major types of PARI. ${ }^{10}{ }^{14}$ Additionally, individual-related indicators, such as age, grade, body mass index, PA level, exercise behaviours and family status, and environmental factors such as weather, exercise facilities and playgrounds, were associated with PARI episodes. ${ }^{911}{ }^{15-18}$ Furthermore, earlier studies revealed that there were marked gender differences in the occurrence and severity of PARI. ${ }^{1015}$ Nevertheless, the evidence of different gender-specific risk factors for PARI is scarce.

In China, students in universities might be more physically active than those in secondary and primary schools due to being free from heavy academic pressure for college admission. ${ }^{10}$ In addition to that, most students have to live in the school dormitory and are therefore independent of their guardians, having more free time to take part in relatively risky activities that were not previously allowed. ${ }^{19}$ This indicates that university students might have a higher susceptibility of sustaining PARI. However, compared with children and adolescents, recent reviews on the problem of PARI in university students noted that epidemiological data on this topic are scant. ${ }^{10} 19$

Collectively, the purpose of this cross-sectional study was to investigate the occurrence of PARI and to explore the gender-specific risk factors associated with injuries resulting from PA participation in Chinese university students via a multicentre survey.

\section{METHODS}

\section{Study participants}

Selected by the method of cluster random sampling, 5628 eligible students graded 1-3 from eight universities (five comprehensive universities and three normal universities) in four Chinese cities (namely, Shantou (Guangdong province), Jinan (Shandong province), Xi'an (Shanxi province) and Nanchang (Jiangxi province)) were invited to participate in the survey during March and April 2017. Informed consent was obtained from the potential study participants, with a response rate of $94.9 \%(n=5341)$. The purpose of this study was orally explained by the authors and the quick response code or hyperlink of the questionnaire was sent to all consenting students.

\section{Data collection}

Wenjuanxing software (Sojump, Changsha, China) was applied as the online platform for the survey. All participants were recruited in nominated classes. The selfadministered online questionnaire was composed of sociodemographic characteristics, PA participation, sleep duration, sedentary behaviour and PARI episodes that occurred in the past 12 months.

Sociodemographic characteristics of the participants included university, study major, grade, gender, age, residence type, any diagnosed chronic disease/symptom (such as heart disease, near-sightedness, hearing disorder and asthma) and sports team membership.

The Global Physical Activity Questionnaire (GPAQ) Chinese version was used to evaluate participants' habitual PA participation during a typical week in the past 12 months. ${ }^{20}$ As one of the most commonly used PA questionnaires, it showed good reliability and validity in the previous study (Spearman's $r=0.81)^{21}$ and sound reliability in our study (Cronbach's $\alpha=0.721$ ). The GPAQ collects the frequency (days per week) and duration (average minutes each day) of moderate-intensity PA (MPA) and vigorous-intensity PA (VPA) in three domains: domestic/ work/study, transportation (MPA only), and sports and leisure-time activities. The PA volume (total cumulative minutes per week) was then calculated by multiplying the frequency and duration of each kind of PA (MPA and VPA) in each domain. Given that the GPAQ does not collect information on specific PAs in which students participated, all students were further asked if they had any favourite PA in which they often took part in the past 12 months. Those with a positive response were required to provide the names of fewer than three activities.

One additional item of time spent in sedentary behaviour was also collected by the GPAQ and the duration (average hours per day) was requested on both a typical weekday and a typical weekend. Furthermore, sleep time (including nap time) was also collected using similar questions. The daily duration in a typical week was then generated and used to classify the participants into five groups based on the average daily sedentary duration (ie, $<4,4$ to $<6,6$ to $<9,9$ to $<12$ and $\geq 12$ hours/day) and sleep time (ie, $<6,6$ to $<7,7$ to $<8,8$ to $<9$ and $\geq 9$ hours/ day).

PARI occurrence in the past 12 months was also collected. The definition of PARI could be found in an earlier study ${ }^{22}$ and a countable PARI episode must have met one or more of the following consequences, which was proven reliably and validly ${ }^{12}$ : the student (1) has to stop the current PA immediately and/or cannot fully participate in the next planned PA and/or (2) cannot go to the school the next day and/or (3) needs to seek medical attention (eg, from providers ranging from first aid personnel to general physicians or physiotherapists). ${ }^{1322}$

\section{Patient and public involvement}

No patients involved.

\section{Statistical analysis}

All statistical analyses were performed by SPSS V.23.0 (SPSS, Inc., Chicago, IL, USA). The person-based incidence rates of PARI were calculated, and the injury risk was calculated as the total number of injuries per number of students per year. ${ }^{23}$ Categorical data were described using numbers and percentages, while continuous data that were normally or not normally distributed were presented as the means and SD or medians and IQR. Pearson $\chi^{2}$ tests and independent-sample $\mathrm{t}$ tests 
or non-parameter tests were used to test between-group differences. All significant variables tested by $\chi^{2}$ tests and independent-sample $t$ tests or non-parameter tests were included together in the multivariable logistic regression model to explore the potential risk factors for PARI occurrence. The ORs and 95\% CIs were calculated with the selection of forward (LR) manner using the criteria of $\alpha_{\text {in }}=0.05$ and $\alpha_{\text {out }}=0.10$. Statistical significance was set at a two-sided $\mathrm{p}<0.05$.

\section{RESULTS}

In total, 5341 students (males: 1790, females: 3551 ) participated in this study, with a mean age of $19.60(\mathrm{SD}=1.27)$. Among the participants, 1293 students (24.2\%) experienced at least one PARI episode in the past 12 months, and male students had a significantly higher injury incidence than females $(26.2 \%(469 / 1790)$ vs $23.2 \%$ (824/3551), $\mathrm{p}=0.016$ ). Overall, 2008 PARI episodes (males: 864, females: 1144) were reported by all injured students. This equals an overall injury risk of 0.38 injuries/student/year, with a significantly higher risk among males than females $(0.48$ vs $0.32, \mathrm{p}<0.05)$.

As presented in table $1, \chi^{2}$ tests revealed significant differences in the injury occurrence by residence type and sports team membership for males (both $\mathrm{p}<0.05$ ). For female students, participating in a sports team, having a disease or symptom, and age affected the occurrence of PARI (all $p<0.05$ ). Irrespective of gender, both males and females in Shantou and Xi'an had a significantly higher incidence of PARI than those in Jinan and Nanchang (both $\mathrm{p}<0.05$ ).

The majority of students participated in transportrelated PA $(79.5 \%)$ and sports and leisure-time PA (58.8\%). Male students with PARI had a significantly higher frequency or longer duration of MPA or VPA in three domains (all $\mathrm{p}<0.01$ ). Similarly, such significant differences could be found between PARI and non-PARI groups for females except for the frequency and duration of transport-related PA (all $\mathrm{p}<0.01$ ). For females, students with different sleep duration significantly differed in

Table 1 Comparison of socio-demographics in participants with PARI or not by gender

\begin{tabular}{|c|c|c|c|c|c|c|}
\hline \multirow[b]{2}{*}{ Characteristics } & \multicolumn{3}{|c|}{ Males $(n=1790)$} & \multicolumn{3}{|c|}{ Females $(n=3551)$} \\
\hline & $\begin{array}{l}\text { PARI } \\
(n=469) \\
n(\%)\end{array}$ & $\begin{array}{l}\text { Non-PARI } \\
(n=1321) \\
n(\%)\end{array}$ & $\chi^{2} / t^{*}$ & $\begin{array}{l}\text { PARI } \\
(n=824) \\
n(\%)\end{array}$ & $\begin{array}{l}\text { Non-PARI } \\
(\mathrm{n}=2727) \\
\mathrm{n}(\%)\end{array}$ & $\chi^{2} / t^{\star}$ \\
\hline City & & & $157.365 \dagger$ & & & $177.880 \dagger$ \\
\hline Shantou & $230(40.4)$ & 339 (59.6) & & $490(31.5)$ & 1064 (68.5) & \\
\hline Jinan & $58(17.2)$ & $279(82.8)$ & & $98(10.5)$ & 832 (89.5) & \\
\hline Xi'an & $76(46.1)$ & 89 (53.9) & & 161 (29.3) & 388 (70.7) & \\
\hline Nanchang & $105(14.6)$ & $614(85.4)$ & & $75(14.5)$ & $443(85.5)$ & \\
\hline Grade & & & 0.873 & & & 0.658 \\
\hline Year 1 & 262 (26.3) & $734(73.7)$ & & $328(23.8)$ & 1050 (76.2) & \\
\hline Year 2 & $126(25.5)$ & $369(74.5)$ & & 261 (22.4) & 902 (77.6) & \\
\hline Year 3 & $81(27.1)$ & 218 (72.9) & & 235 (23.3) & 775 (76.7) & \\
\hline Residence type & & & $6.886 \dagger$ & & & 0.249 \\
\hline Dormitory & $325(25.8)$ & $935(74.2)$ & & 567 (22.6) & $1943(77.4)$ & \\
\hline Home & $142(28.3)$ & $359(71.7)$ & & 244 (24.6) & 746 (75.4) & \\
\hline Other & $2(6.9)$ & $27(93.1)$ & & $13(25.5)$ & $38(74.5)$ & \\
\hline Sports team member & & & $39.753 \S$ & & & $37.292 \S$ \\
\hline No & $354(23.4)$ & 1159 (76.6) & & $697(21.8)$ & 2504 (78.2) & \\
\hline Yes & $115(41.5)$ & $162(58.5)$ & & 127 (36.3) & $223(63.7)$ & \\
\hline Chronic disease/symptc & & & 1.703 & & & $12.111 \neq$ \\
\hline No & $441(26.6)$ & $1218(73.4)$ & & 767 (22.7) & 2618 (77.3) & \\
\hline Yes & $28(21.4)$ & $103(78.6)$ & & 57 (34.3) & $109(65.7)$ & \\
\hline Age $(\overline{\mathrm{x} \pm \mathrm{S}}$, years $)$ & $19.52 \pm 1.30$ & $19.40 \pm 1.32$ & 1.857 & $19.77 \pm 1.27$ & $19.66 \pm 1.27$ & $2.228 \dagger$ \\
\hline
\end{tabular}

${ }^{*}$ Categorical variables (all variables except for age) were tested by Pearson $\chi^{2}$ tests, and continuous variables (ie, age) were tested by independent-sample t tests.

$\uparrow \mathrm{p}<0.05$.

$\neq \mathrm{p}<0.01$.

$\S p<0.001$.

PARI, physical activity-related injury. 
Table 2 Comparison of lifestyle variables in participants with PARI or not among different genders

\begin{tabular}{|c|c|c|c|c|c|c|}
\hline \multirow[b]{2}{*}{ Characteristics } & \multicolumn{3}{|c|}{ Males $(n=1790)$} & \multicolumn{3}{|c|}{ Females $(n=3551)$} \\
\hline & $\begin{array}{l}\text { PARI } \\
(\mathrm{n}=469) \\
\text { median, IQR }\end{array}$ & $\begin{array}{l}\text { Non-PARI } \\
(n=1321) \\
\text { median, IQR }\end{array}$ & $\chi^{2} / Z^{*}$ & $\begin{array}{l}\text { PARI } \\
\text { ( } n=824) \\
\text { median, IQR }\end{array}$ & $\begin{array}{l}\text { Non-PARI } \\
(n=2727) \\
\text { median, IQR }\end{array}$ & $\chi^{2} / Z^{*}$ \\
\hline \multicolumn{7}{|l|}{ Domestic/work/study } \\
\hline \multicolumn{7}{|l|}{ VPA } \\
\hline Frequency, day/week & $1(0,2)$ & $0(0,1)$ & $5.392 \S$ & $0(0,0.75)$ & $0(0,0)$ & $4.624 \S$ \\
\hline Duration, min/day & $10(0,30)$ & $0(0,15)$ & $5.767 \S$ & $0(0,7.5)$ & $0(0,0)$ & $4.818 \S$ \\
\hline \multicolumn{7}{|l|}{ MPA } \\
\hline Frequency, day/week & $2(0,3)$ & $1(0,3)$ & $4.235 \S$ & $1(0,3)$ & $0(0,2)$ & $5.197 \S$ \\
\hline Duration, min/day & $30(0,40)$ & $12(0,30)$ & $6.593 \S$ & $10(0,30)$ & $0(0,30)$ & $5.341 \S$ \\
\hline \multicolumn{7}{|l|}{ Transportation } \\
\hline Frequency, day/week & $5(0,7)$ & $5(0,7)$ & $2.998 \ddagger$ & $5(2,7)$ & $5(1,7)$ & 1.816 \\
\hline Duration, min/day & $30(15,60)$ & $30(0,45)$ & $4.156 \S$ & $30(12.75,45)$ & $30(10,45)$ & 0.697 \\
\hline \multicolumn{7}{|l|}{ Sports and leisure time } \\
\hline \multicolumn{7}{|l|}{ VPA } \\
\hline Frequency, day/week & $1(0,2)$ & $0(0,1)$ & $9.538 \S$ & $0(0,1)$ & $0(0,1)$ & $3.130 \ddagger$ \\
\hline Duration, min/day & $20(0,60)$ & $0(0,20)$ & $10.402 \S$ & $0(0,20)$ & $0(0,10)$ & $3.050 \ddagger$ \\
\hline \multicolumn{7}{|l|}{ MPA } \\
\hline Frequency, day/week & $2(0,3)$ & $1(0,2)$ & $5.545 \S$ & $1(0,2)$ & $0(0,2)$ & $5.644 \S$ \\
\hline Duration, min/day & $20(0,40)$ & $10(0,30)$ & $7.235 \S$ & $10(0,30)$ & $0(0,30)$ & $5.485 \S$ \\
\hline Sleep duration, n (\%) & & & 4.055 & & & $13.345 \dagger$ \\
\hline$<6$ hours/day & $33(20.4)$ & $129(79.6)$ & & $61(27.0)$ & $165(73.0)$ & \\
\hline 6 to $<7$ hours/day & $93(27.2)$ & $249(72.8)$ & & $145(28.2)$ & $370(71.8)$ & \\
\hline 7 to $<8$ hours/day & $184(26.4)$ & $513(73.6)$ & & $305(20.9)$ & $1151(79.1)$ & \\
\hline 8 to $<9$ hours/day & $126(27.9)$ & $325(72.1)$ & & $225(22.8)$ & 764 (77.2) & \\
\hline$\geq 9$ hours/day & $33(24.1)$ & $104(75.9)$ & & $88(24.1)$ & $277(75.9)$ & \\
\hline \multicolumn{2}{|c|}{ Sedentary behaviour, n (\%) } & & $18.567 \ddagger$ & & & 7.094 \\
\hline$<4$ hours/day & $173(21.3)$ & $638(78.7)$ & & $269(24.0)$ & $853(76.0)$ & \\
\hline 4 to $<6$ hours/day & $42(28.4)$ & $106(71.6)$ & & $72(28.9)$ & $177(71.1)$ & \\
\hline 6 to $<9$ hours/day & $40(30.3)$ & $92(69.7)$ & & $71(24.0)$ & $225(76.0)$ & \\
\hline 9 to $<12$ hours/day & $62(30.0)$ & $145(70.0)$ & & $89(20.1)$ & 354 (79.9) & \\
\hline$\geq 12$ hours/day & $152(30.9)$ & $340(69.1)$ & & $323(22.4)$ & 1118 (77.6) & \\
\hline
\end{tabular}

${ }^{*}$ Categorical variables (ie, sleep duration and sedentary behaviour) were tested by Pearson $\chi^{2}$ tests, and continuous variables (except for sleep duration and sedentary behaviour) were tested by Mann-Whitney tests or independent-sample $t$ tests (the frequency of transport-related PA). $\mathrm{tp}<0.05$.

$\neq \mathrm{p}<0.01$.

$\S p<0.001$

MPA, moderate-intensity physical activity; PA, physical activity; PARI, physical activity-related injury; VPA, vigorous-intensity physical activity.

PARI experience $(\mathrm{p}<0.05)$, while sedentary behaviour had an impact on the occurrence of PARI among male students $(\mathrm{p}<0.01)$ (table 2). The means and SDs for all PA indicators are available in online supplemental table 1 .

Based on the significant variables tested by $\chi^{2}$ tests and independent-sample t tests or Mann-Whitney tests, a multivariable logistic regression model was used to estimate ORs and corresponding 95\% CIs for PARI among male students. As shown in table 3, male students in Shantou and Xi'an had higher odds of sustaining PARI
$(\mathrm{OR}=3.525$ and 4.571). Sports team members were more likely to suffer from PARI (OR=1.819, 95\% CI 1.349 to 2.453 ) in comparison with their counterparts. Additionally, the frequency and duration of sports and leisure-time VPA were significantly associated with PARI ( $\mathrm{OR}=1.129$ and 1.007, respectively).

Similarly, a multivariable logistic regression analysis was also performed to estimate the odds of potential factors for PARI among females, and the results of all significant variables kept in the final model are displayed in 


\begin{tabular}{|c|c|c|c|c|}
\hline B & SE & OR & $95 \% \mathrm{Cl}$ & $p$ value \\
\hline \multicolumn{5}{|c|}{ City } \\
\hline-0.060 & 0.186 & 0.941 & 0.653 to 1.356 & 0.745 \\
\hline 1.520 & 0.194 & 4.571 & 3.124 to 6.687 & $<0.001$ \\
\hline \multicolumn{5}{|c|}{ Sports team member } \\
\hline & & 1 (ref.) & & \\
\hline 0.598 & 0.152 & 1.819 & 1.349 to 2.453 & $<0.001$ \\
\hline \multicolumn{5}{|c|}{ Sports and leisure-time VPA } \\
\hline 0.121 & 0.040 & 1.129 & 1.043 to 1.221 & 0.003 \\
\hline
\end{tabular}

PARI, physical activity-related injury; VPA, vigorous-intensity physical activity.

table 4. Female students in Shantou and Xi'an were more vulnerable to experiencing PARI $(\mathrm{OR}=2.710$ and 2.456, respectively), and those who participated in a sports team and had a chronic condition were more prone to sustain PARI (OR=1.950 and 1.834, respectively). Moreover, a higher frequency of sports and leisure-time VPA and MPA $(\mathrm{OR}=1.079$ and 1.091 , respectively) and longer duration of sports and leisure-time MPA $(\mathrm{OR}=1.003)$ increased the possibility of suffering from PARI.

With regard to favourite activities, nearly three-fifths $(58.9 \%, \mathrm{n}=3147)$ of students had at least one PA that they liked and participated often during the past 12 month. Running, badminton, basketball, bicycling, table tennis and swimming were the primary activities in which students participated in the past 12 months. There was a difference in PA engagement. Males $(n=1107)$ favoured running, basketball, badminton, table tennis, bicycling and football, while females $(\mathrm{n}=2040)$ preferred running, badminton, bicycling, basketball, table tennis and swimming (see online supplemental table 2).

The number and consequences of PARI reported by 1293 injured students before and after gender stratification are shown in table 5 . During the past 12 months, more than half $(n=666,51.5 \%)$ of the injured participants experienced one PARI episode and over one-fifth $(\mathrm{n}=296,22.9 \%)$ suffered from PARI at least three times (ie, multiple injuries). Male students had a greater tendency to sustain multiple injuries, whereas a larger

\begin{tabular}{|c|c|c|c|c|c|}
\hline Variables & B & SE & OR & $95 \% \mathrm{Cl}$ & $P$ value \\
\hline \multicolumn{6}{|l|}{ City } \\
\hline Shantou & 0.997 & 0.139 & 2.710 & 2.064 to 3.559 & $<0.001$ \\
\hline Jinan & -0.494 & 0.169 & 0.868 & 0.674 to 1.092 & 0.355 \\
\hline Xi'an & 0.898 & 0.159 & 2.456 & 1.796 to 3.357 & $<0.001$ \\
\hline Nanchang & & & 1 (ref.) & & \\
\hline \multicolumn{6}{|l|}{ Sports team member } \\
\hline No & & & 1 (ref.) & & \\
\hline Yes & 0.668 & 0.128 & 1.950 & 1.516 to 2.507 & $<0.001$ \\
\hline \multicolumn{6}{|l|}{ Chronic disease/symptom } \\
\hline \multicolumn{6}{|l|}{ No } \\
\hline Yes & 0.607 & 0.178 & 1.834 & 1.293 to 2.602 & 0.001 \\
\hline \multicolumn{6}{|c|}{ Sports and leisure-time VPA } \\
\hline Frequency, day/week & 0.076 & 0.034 & 1.079 & 1.011 to 1.153 & 0.034 \\
\hline \multicolumn{6}{|c|}{ Sports and leisure-time MPA } \\
\hline Frequency, day/week & 0.087 & 0.026 & 1.091 & 1.036 to 1.149 & 0.001 \\
\hline Duration, min/day & 0.003 & 0.001 & 1.003 & 1.001 to 1.006 & 0.016 \\
\hline
\end{tabular}

PARI, physical activity-related injury; VPA, vigorous-intensity physical activity; MPA, moderate-intensity physical activity. 
Table 5 Number and consequences of PARI among injured university students

\begin{tabular}{|c|c|c|c|c|c|}
\hline Characteristics & Total (N=1293), n (\%) & Males (N=469), n (\%) & Females ( $\mathrm{N}=824), \mathrm{n}(\%)$ & $\chi^{2}$ & $p$ value \\
\hline 1 & $666(51.5)$ & $198(42.2)$ & $468(56.8)$ & & \\
\hline$\geq 3$ & $296(22.9)$ & $147(31.3)$ & $149(18.1)$ & & \\
\hline \multicolumn{6}{|c|}{ Consequences of PARI } \\
\hline No & $552(42.7)$ & $168(35.8)$ & $384(46.6))$ & & \\
\hline Yes & 741 (57.3) & $301(64.2)$ & $440(53.4)$ & & \\
\hline \multicolumn{4}{|l|}{ Absence of class } & 0.306 & 0.580 \\
\hline No & $984(76.1)$ & $361(77.0)$ & $623(75.8)$ & & \\
\hline Yes & $512(39.6)$ & 175 (27.3) & 337 (40.9) & & \\
\hline
\end{tabular}

PARI, physical activity-related injury; PA, physical activity.

portion of females experienced PARI only once. Over half of the injured $(57.3 \%)$ experienced a withdrawal time of PA and nearly two-fifths (39.6\%) received medical treatment due to PARI. A significant difference was observed for both genders in a break from PA participation, with male injured students being more likely to experience inactivity $(64.2 \%$ vs $53.4 \%$, p $<0.001)$.

\section{DISCUSSION}

As one of the top health threats to school-aged adolescents and young adults in the majority of countries, ${ }^{5} 11$ PARI could lead to financial medical burdens and prevent individuals from being physically active to improve and maintain physical, cognitive and mental health. ${ }^{24-26}$ In this study, we found that approximately one out of four university students experienced at least one PARI episode in the past 12 months, with an overall injury risk of 0.38 injuries/student/year. Additionally, more than half of the injured students had a withdrawal time of PA participation, and nearly two-fifths of cases required medical attention. These findings indicated that PARI is rather common and has a great adverse effect on Chinese university students. In contemporary China, approximately $48 \%$ of secondary students could be admitted to different levels of universities. ${ }^{27}$ The $1 \%$ National Sample Census in 2015 revealed that the number of current university students hit 39.7 million. ${ }^{27}$ This suggests that the problem of PARI among Chinese university students needs to be urgently highlighted, and effective injury-prevention programmes should be developed when PA promotion is made a public health priority.

In line with other findings reported elsewhere, ${ }^{23} 2829$ we found that male students had a significantly higher PARI incidence and risk than their female counterparts.
Several reasons may underline this gender difference. First, males are prone to be more physically active than females. ${ }^{30}$ This could be supported by our data in terms of different PA indicators in table 2. Second, Ristolainen $e t a l^{31}$ and Hootman $e t a l^{2}$ revealed that males are inclined to take part in more competitive high-intensity activities, such as basketball and football, that involve a higher rate of contact, jumping and sprinting, which are commonly associated with a higher incidence of injuries. Though the GPAQ could not allow us to access the type of PA, our study found differences in favourite activities by gender-more male students favoured basketball and football. This might support the relationship between the intensity and type of PA to a certain extent. Even in the same activity, males tend to have higher competitiveness and resistance with lower individual safety awareness. ${ }^{33}$ This could contribute to their higher incidence of PARI to some extent. Third, Deci and Ryan ${ }^{34}$ indicated that male students had great motivation, impulsiveness and self-determination, which may also play a role. On one hand, these gender-specific characteristics could affect the occurrence of PARI; on the other hand, these might influence the analysis of potential risk factors for PARI. Previous studies have revealed marked genderspecific differences in PARI occurrence and relevant risk factors. ${ }^{153536}$ If a risk factor has different impacts on PARI for various genders, it would be difficult to find true associations when the data were analysed together. ${ }^{35} \mathrm{We}$, thus, explored possible contributors to PARI for males and females separately.

Being physically inactive is harmful to our individual well-being, ${ }^{5}$ but so is being too active. Our study revealed a positive and significant association between PARI occurrence and the frequency of sports and leisure-time VPA 
participation in both males and females, and a longer duration of sports and leisure-time VPA involvement could increase the risk of PARI for male students. This is highly consistent with other findings showing that higher intensity, higher frequency and longer duration of PA participation contributed to an elevated risk of PARI. ${ }^{33} 37$ Furthermore, we found that the frequency and duration of sports and leisure-time MPA engagement were positively associated with the occurrence of PARI for females. Collectively, different levels of PA participation between males and females cause differences in the occurrence of PARI. This is in line with other reports. ${ }^{35} 36$ In addition, though a relationship between sedentary behaviour and PARI occurrence was not observed in our study, earlier literature indicated that sedentary behaviour would affect individual physical function and increase the risk of injury. ${ }^{38-40}$ We should place great emphasis on the above results, especially when we promote a physically active lifestyle for the public. Otherwise, the benefits of PA participation would be comprised.

In comparison with their counterparts, sports team members had a higher likelihood of suffering from PARI among both males $(\mathrm{OR}=1.819)$ and females $(\mathrm{OR}=1.950)$. This parallels previous literature, ${ }^{9}{ }^{41}$ irrespective of age group. Generally, students participating in sports teams would spend lots of their time on one certain kind of activity, which often associates with the high risk of overuse injuries, such as tendinopathies, bursitis and stress fracture. $^{42}{ }^{43}$ Moreover, the activities in which sports team members participate are usually structured, and their focus is often on improving individual performance. ${ }^{41}$ In this way, they generally have a higher frequency and intensity and longer duration of PA participation. ${ }^{41}$ This further supports the idea that too much PA can harm individual health. Thus, we should take this particularly vulnerable population into consideration, and effective preventative measures should be introduced.

In this study, living with a chronic condition was identified as the potential determinant of PARI for females. Among the whole study sample, nearly $5.7 \%$ had a chronic disease (ie, near-sightedness). The exact reasons for the association between the chronic condition and PARI occurrence in female students in the present study are unknown. A possible partial explanation may be that the health issue affects their PA participation and exposes them to higher injury risk when undertaking PA. This specific contributor has implications for the identification of injury mechanisms and interventions in injury prevention among female students.

Surprisingly, our study revealed a difference in the occurrence of PARI among university students-both males and females in Shantou and Xi' an had markedly higher PARI incidence than those in Jinan and Nanchang. However, this discrepancy could not be explained by all the potential variables in our study (see online supplemental tables 3 and 4). Given that the four study cities are located in different parts of China and have varied climates, we assumed that the large between-city differences in the incidence rate of PARI may be attributed to geographical factors. PA infrastructure may affect the occurrence of PARI for students, but all these study cities belong to Mainland China, sharing similar facilities and surveillance systems. We thereby excluded this possibility. Additionally, the urban environments outside of the universities might be attributable to this difference, but we could not provide related evidence for this hypothesis due to the absence of data on where these injuries occurred. Collectively, the reasons for the between-city PARI difference need to be studied further.

Our study is influenced by several limitations. First, data collection was through a structured self-reported questionnaire, which could lead to reporting bias and recall bias. ${ }^{44}$ For example, students might not have accurately reported their PARI experiences, in particular the minor and earliest injuries. Though previous studies noted that participants were able to correctly indicate whether they had been injured or not during the past 12 -month period, ${ }^{45}$ we should consider a shorter recall period, that is, 6months, especially when collecting detailed information of each identified PARI episode. Meanwhile, despite the good reliability and validity of the GPAQ we could not fully preclude the possibility of over-reported PA exposure time. Despite this drawback, the use of self-report is a more practical, feasible, and cost-effective way that allows us to carry out a multicentre survey with such a large sample size. Second, the nature of the cross-sectional study limited us from drawing the cause-and-effect relationship between PARI outcome and the potential risk factors in this study. Their associations still warrant further investigation by a prospective cohort study. Third, nearly two-thirds of the study samples were female students, which may have limited the generalisability and representativeness of our findings. Fourth, we did not compare acute and overuse injuries, two injury types with different injury mechanisms, which may have deviated the analysis in the association between PARI and its potential contributors. Thus, future studies should take these limitations into account to better reflect the characteristics and risk factors of PARI among university students.

\section{CONCLUSION}

With an overall incidence rate of $24.2 \%$ and an injury risk of 0.38 injuries/student/year, PARI was not uncommon among Chinese university students. Different genders differed in the occurrence of PARI and were affected by various potential risk factors. For males, those who were Shantou and Xi'an students, were sports team members, and participated in sports and leisure-time VPA with higher frequency and longer duration were more likely to suffer from PARI. For females, studying in Shantou and Xi'an, participating in a sports team, having a chronic disease or symptom, engaging in sports and leisure-time VPA with higher frequency, and taking part in sports and leisure-time MPA with higher frequency and longer duration were the potential contributors to PARI. These findings provide a direction to develop targeted genderspecific prophylactic interventions to reduce PARI and to 
maximise the benefits of PA participation among university students in China.

\section{Author affiliations}

${ }^{1}$ Injury Prevention Research Center, Shantou University Medical College, Shantou, China

${ }^{2}$ School of Public Health, Shantou Univeristy, Shantou, China

${ }^{3}$ Shenzhen Center for Chronic Disease Control, Shenzhen, China

${ }^{4}$ School of Nursing, Xi'an Innovation College of Yan'an University, Xi'an, China

${ }^{5}$ School of Nursing \& Optometry, Jiangxi Teachers College, Yingtan, China

${ }^{6}$ Department of Epidemiology, Shandong University School of Public Health, Jinan, China

${ }^{7}$ Department of Sport, Physical Education and Health, Hong Kong Baptist University, Hong Kong, China

${ }^{8}$ Centre for Health and Exercise Science Research, Hong Kong Baptist University, Hong Kong, China

Correction notice This article has been corrected since it first published. The provenance and peer review statement has been included.

Acknowledgements We thank all university students for their participation in our questionnaire survey.

Contributors LL, CJ and YG conceived and designed the study. WC, PY, XY, LG and WY collected data; and WC and SC performed the statistical analyses and drafted the manuscript. All authors read and approved the final version of the manuscript, and agreed with the order of the presentation of the authors.

Funding This work was supported by the National Natural Science Foundation of China (grant number 31640038).

Disclaimer The sponsor had no role in the study design, data collection, analysis and interpretation, preparation and revision of the manuscript, or decision to submit the article for publication.

Competing interests None declared.

Patient consent for publication Not required.

Ethics approval This study was strictly carried out according to the Declaration of Helsinki and approved by the (Shantou University Medical College Ethics Committee (SUMC-2016-22), Xi'an Innovation College of Yan'an University Ethics Committee, Jiangxi Teachers College Ethics Committee, and Shandong University Ethics Committee (20161101).

Provenance and peer review Not commissioned; externally peer reviewed. Data availability statement № additional data available.

Supplemental material This content has been supplied by the author(s). It has not been vetted by BMJ Publishing Group Limited (BMJ) and may not have been peer-reviewed. Any opinions or recommendations discussed are solely those of the author(s) and are not endorsed by BMJ. BMJ disclaims all liability and responsibility arising from any reliance placed on the content. Where the content includes any translated material, BMJ does not warrant the accuracy and reliability of the translations (including but not limited to local regulations, clinical guidelines, terminology, drug names and drug dosages), and is not responsible for any error and/or omissions arising from translation and adaptation or otherwise.

Open access This is an open access article distributed in accordance with the Creative Commons Attribution Non Commercial (CC BY-NC 4.0) license, which permits others to distribute, remix, adapt, build upon this work non-commercially, and license their derivative works on different terms, provided the original work is properly cited, appropriate credit is given, any changes made indicated, and the use is non-commercial. See: http://creativecommons.org/licenses/by-nc/4.0/.

ORCID iDs

Weicong Cai http://orcid.org/0000-0002-1597-4313

Yang Gao http://orcid.org/0000-0003-0439-4804

\section{REFERENCES}

1 ISPAH International Society for Physical Activity and Health. The Bangkok declaration on physical activity for global health and sustainable development. Br J Sports Med 2017;51:1389-91.

2 Warburton DE, Charlesworth S, Ivey A, et al. A systematic review of the evidence for Canada's physical activity guidelines for adults. Int $J$ Behav Nutr Phys Act 2010;7:39.
3 Garber CE, Blissmer B, Deschenes MR, et al. Quantity and quality of exercise for developing and maintaining cardiorespiratory, musculoskeletal, and neuromotor fitness in apparently healthy adults. Med Sci Sports Exerc 2011:43:1334-59.

4 WHO. Global action plan for the prevention and control of noncommunicable diseases 2013-2020. Geneva: World Health Organization, 2013. https://www.who.int/nmh/events/ncd_action plan/en/

5 WHO. Global recommendations on physical activity for health. Geneva: World Health Organization, 2010. https://www.who.int/ dietphysicalactivity/publications/9789241599979/en/

6 Education CN. Suggestion of CPC Central Committee and State Council on strengthing the youth sports to enhance youth physical [in Chinese]. Chin J Sch Health 2007;28:481-3.

7 Haskell WL, Lee I-M, Pate RR, et al. Physical activity and public health: updated recommendation for adults from the American College of sports medicine and the American heart association. Circulation 2007;116:1081-93.

8 Frisch A, Seil R, Urhausen A, et al. Analysis of sex-specific injury patterns and risk factors in young high-level athletes. Scand J Med Sci Sports 2009;19:834-41.

9 Cai W, Gao Y, Yang W, et al. Physical activity-related injury and its associated factors among middle school students in southern China. Int J Environ Res Public Health 2018;15:e1244.

10 Cai W, Gao L, Li L, et al. Epidemiology of physical activity-related injuries in Chinese university students. Scand J Med Sci Sports 2019;29:1331-9.

11 Pickett W, Molcho M, Simpson K, et al. Cross national study of injury and social determinants in adolescents. Inj Prev 2005;11:213-8.

12 van Mechelen W, Verhagen E. Essay: injury prevention in young people--time to accept responsibility. Lancet 2005;366 Suppl 1:S46.

13 van Mechelen W, Hlobil H, Kemper HC. Incidence, severity, aetiology and prevention of sports injuries. A review of concepts. Sports Med 1992;14:82-99.

14 Zuckerman SL, Wegner AM, Roos KG, et al. Injuries sustained in national collegiate athletic association men's and women's basketball, 2009/2010-2014/2015. Br J Sports Med 2018;52:261-8.

15 Grimmer KA, Jones D, Williams J. Prevalence of adolescent injury from recreational exercise: an Australian perspective. J Adolesc Health 2000;27:266-72.

16 Costa E Silva L, Fragoso MI, Teles J. Physical activity-related injury profile in children and adolescents according to their age, maturation, and level of sports participation. Sports Health 2017;9:118-25.

17 Richmond SA, Kang J, Emery CA. Is body mass index a risk factor for sport injury in adolescents? J Sci Med Sport 2013;16:401-5.

18 Gamage PJ, Fortington LV, Finch CF. Perceived injury risk among junior cricketers: a cross sectional survey. Int $J$ Environ Res Public Health 2017;14:e946.

19 Hubbard-Turner T, Turner MJ. Physical activity levels in college students with chronic ankle instability. J Athl Train 2015;50:742-7.

20 WHO. Chroic diseases and health promotion. global physical activity surveillance, 2017. Available: http://www.who.int/ncds/surveillance/ steps/GPAQ/en/

21 Bull FC, Maslin TS, Armstrong T. Global physical activity questionnaire (GPAQ): nine country reliability and validity study. $J$ Phys Act Health 2009;6:790-804.

22 Bloemers F, Collard D, Paw MCA, et al. Physical inactivity is a risk factor for physical activity-related injuries in children. $\mathrm{Br} J$ Sports Med 2012;46:669-74.

23 Goossens L, Verrelst R, Cardon G, et al. Sports injuries in physical education teacher education students. Scand J Med Sci Sports 2014:24:683-91.

24 Collard DCM, Verhagen EALM, van Mechelen W, et al. Economic burden of physical activity-related injuries in Dutch children aged 1012. Br J Sports Med 2011;45:1058-63.

25 Finch $\mathrm{C}$, Cassell E. The public health impact of injury during sport and active recreation. J Sci Med Sport 2006;9:490-7.

26 Finch C, Owen N, Price R. Current injury or disability as a barrier to being more physically active. Med Sci Sports Exerc 2001;33:778-82.

27 Wang G. The size, structure and developing trend of the Chinese college-age population [in Chinese]. Popul Econ 2017;6:83-93.

28 Gutierrez G, Sills M, Bublitz CD, et al. Sports-Related injuries in the United States: who gets care and who does not. Clin J Sport Med 2006;16:136-41.

29 Gilchrist J, Haileyesus T, Murphy MW, et al. Nonfatal sports and recreation heat illness treated in hospital emergency departments-United States, 2001-2009. MMWR Morb Mortal Wkly Rep 2011:60:977-80.

30 Hallal PC, Andersen LB, Bull FC, et al. Global physical activity levels: surveillance progress, pitfalls, and prospects. Lancet 2012;380:247-57. 
31 Ristolainen L, Heinonen A, Turunen $\mathrm{H}$, et al. Type of sport is related to injury profile: a study on cross country skiers, swimmers, longdistance runners and soccer players. A retrospective 12-month study. Scand J Med Sci Sports 2010;20:384-93.

32 Hootman JM, Dick R, Agel J. Epidemiology of collegiate injuries for 15 sports: summary and recommendations for injury prevention initiatives. J Athl Train 2007;42:311-9.

33 Pons-Villanueva J, Seguí-Gómez M, Martínez-González MA. Risk of injury according to participation in specific physical activities: a 6-year follow-up of 14356 participants of the sun cohort. Int J Epidemiol 2010;39:580-7.

34 Deci EL, Ryan RM. Intrinsic motivation and Self-Determination. Encycl Appl Psychol 2004;3:437-48.

35 Tang D, Cai W, Yang W, et al. Gender-Specific risk factors of physical activity-related injuries among middle school students in southern China. Int J Environ Res Public Health 2019;16:e2359.

36 Cai W, Sun Y, Peng K, et al. Physical-Activity-Related injuries and risk factors among secondary school students in Hong Kong. Int $J$ Environ Res Public Health 2020;17:e747.

37 Parkkari J, Kannus P, Natri A, et al. Active living and injury risk. Int J Sports Med 2004;25:209-16.
38 Peterson NE, Sirard JR, Kulbok PA, et al. Sedentary behavior and physical activity of young adult university students. Res Nurs Health 2018;41:30-8.

39 LaMonte MJ, Wactawski-Wende J, Larson JC, et al. Association of physical activity and fracture risk among postmenopausal women. JAMA Netw Open 2019;2:e1914084.

40 Garstang SV, Stitik TP. Osteoarthritis: epidemiology, risk factors, and pathophysiology. Am J Phys Med Rehabil 2006;85:S2-11-quiz S1214.

41 Räisänen AM, Parkkari J, Karhola L, et al. Adolescent physical activity-related injuries in sports Club, school sports and other leisure time physical activities. Cogent Medicine 2016;3:1260786.

42 Wilder RP, Sethi S. Overuse injuries: tendinopathies, stress fractures, compartment syndrome, and shin splints. Clin Sports Med 2004;23:55-81.

43 Yang J, Tibbetts AS, Covassin T, et al. Epidemiology of overuse and acute injuries among competitive collegiate athletes. J Athl Train 2012;47:198-204.

44 Ara I, Aparicio-Ugarriza R, Morales-Barco D, et al. Physical activity assessment in the general population; validated self-report methods. Nutr Hosp 2015;31 Suppl 3:211-8.

45 Gabbe BJ, Finch CF, Bennell KL, et al. How valid is a self reported 12 month sports injury history? Br J Sports Med 2003;37:545-7. 